\title{
Natural constructions of some generalized Kac-Moody algebras as bosonic strings
}

\author{
Thomas Creutzig, Alexander Klauer and \\ NiLs R. ScheithaueR
}

\begin{abstract}
There are 10 generalized Kac-Moody algebras whose denominator identities are completely reflective automorphic products of singular weight on lattices of squarefree level. Under the assumption that the meromorphic vertex operator algebra of central charge 24 and spin-1 algebra $\hat{A}_{p-1, p}^{r}$ exists we show that four of them can be constructed in a uniform way from bosonic strings moving on suitable target spaces.
\end{abstract}

\section{Introduction}

Generalized Kac-Moody algebras are natural generalizations of the finitedimensional simple Lie algebras. They are defined by generators and relations and are allowed to have imaginary simple roots. Generalized Kac-Moody algebras are in general infinite-dimensional but their theory is in many aspects similar to the finite-dimensional theory. In particular there is a character formula for highest weight modules and a denominator identity. Borcherds has used twisted versions of the denominator identity of the monster algebra to prove Conway and Norton's moonshine conjecture [3].

Borcherds' singular theta correspondence [5] is a map from modular forms for the Weil representation to automorphic forms on orthogonal groups. Since these automorphic forms can be written as infinite products they are called automorphic products. They have found various applications in geometry, arithmetic and in the theory of Lie algebras. In particular, the denominator identities of generalized Kac-Moody algebras are sometimes automorphic products (cf. $[18,19])$. Reflective automorphic products are automorphic products whose divisors correspond to roots of the underlying lattice and are zeros of order 1 . They can be classified under certain conditions [20].

In [18], a family of 10 generalized Kac-Moody algebras is constructed. Recall that the Mathieu group $M_{23}$ acts on the Leech lattice $\Lambda$. Let $g$ be an 
element of squarefree order $N$ in $M_{23}$. Then $g$ has characteristic polynomial $\prod_{k \mid N}\left(x^{k}-1\right)^{24 / \sigma_{1}(N)}$ as automorphism of $\Lambda$. The eta product

$$
\eta_{g}(\tau)=\prod_{k \mid N} \eta(k \tau)^{24 / \sigma_{1}(N)}
$$

is a cusp form for $\Gamma_{0}(N)$ with multiplicative coefficients. The fixpoint lattice $\Lambda^{g}$ is the unique lattice in its genus without roots. We lift $f_{g}=1 / \eta_{g}$ to a vector valued modular form

$$
F_{g}=\left.\sum_{M \in \Gamma_{0}(N) \backslash \Gamma} f_{g}\right|_{M} \rho_{D}\left(M^{-1}\right) e^{0}
$$

for the Weil representation $\rho_{D}$ of the lattice $\Lambda^{g} \oplus I I_{1,1} \oplus \sqrt{N} I_{1,1}$. Then we apply the singular theta correspondence to $F_{g}$ to obtain an automorphic product $\Psi_{g}$ (cf. [3, Theorem 13.3]). We summarize this in the following diagram

$$
g \mapsto \frac{1}{\eta_{g}} \mapsto F_{g} \mapsto \Psi_{g}
$$

The automorphic form $\Psi_{g}$ has singular weight and is completely reflective, i.e., has zeros of order 1 corresponding to all roots of $\Lambda^{g} \oplus I I_{1,1} \oplus \sqrt{N} I I_{1,1}$ (cf. [20]). The expansion of $\Psi_{g}$ at any cusp is given by

$$
e^{\rho} \prod_{d \mid N} \prod_{\alpha \in\left(L \cap d L^{\prime}\right)^{+}}\left(1-e^{\alpha}\right)^{\left[1 / \eta_{g}\right]\left(-\alpha^{2} / 2 d\right)}=\sum_{w \in W} \operatorname{det}(w) w\left(\eta_{g}\left(e^{\rho}\right)\right)
$$

where $L=\Lambda^{g} \oplus I I_{1,1}, \rho$ is a primitive norm 0 vector in $I I_{1,1}$ and $W$ is the full reflection group of $L$. This is the denominator identity of a generalized KacMoody algebra whose real simple roots are the simple roots of $W$, i.e., the roots $\alpha$ of $L$ with $(\rho, \alpha)=-\alpha^{2} / 2$, and imaginary simple roots are the positive multiples $n \rho$ of the Weyl vector with multiplicity $24 \sigma_{0}((N, n)) / \sigma_{1}(N)$.

In the prime order case, the above Lie algebras have also been constructed by a different method in [17] (cf. also [3, Section 14]).

One of the main results of [20] is that the 10 generalized Kac-Moody algebras corresponding to the elements of squarefree order in $M_{23}$ are the only generalized Kac-Moody algebras whose denominator identities are completely reflective automorphic products of singular weight on lattices of squarefree level splitting two hyperbolic planes.

For $N=1$ and 2 these Lie algebras represent the physical states of bosonic strings moving on suitable spacetimes (cf. $[2,13]$ ). 
Let $V$ be a meromorphic vertex operator algebra of central charge 24 and nonzero $L_{0}$-eigenspace $V_{1}$. Schellekens [21] proves that either $V_{1}$ has dimension 24 and $V$ is the vertex operator algebra of the Leech lattice or $V_{1}$ has dimension greater than 24 . He shows that in this case there are exactly 69 modular invariant partition functions and describes explicitly the corresponding vector spaces as sums of highest weight modules over affine Kac-Moody algebras. If the monster vertex operator algebra is unique and for each of the 69 partition functions there exists a unique vertex operator algebra, Schellekens' result implies that there are 71 meromorphic vertex operator algebras of central charge 24 . Up to now these conjectures are open.

In this paper we prove the following results.

Let $p=2,3,5$ or 7 and let $V$ be the prospective vertex operator algebra $V$ in [21] of central charge 24, trivial fusion algebra and spin-1 algebra $\hat{A}_{q, p}^{r}$, where $q=p-1$ and $r=48 / q(p+1)$. Then the character of $V$ as $\hat{A}_{q}^{r}$-module can be written as

$$
\chi=\sum_{\gamma \in N^{\prime} / N} F_{\gamma} \vartheta_{\gamma}
$$

Here $N$ is the unique lattice of minimal norm 4 for $p=2,3,5$ and minimal norm 6 for $p=7$ in the genus

$$
I I_{2 m, 0}\left(p^{\epsilon_{p}(m+2)}\right)
$$

where $m=24 /(p+1)$ and $\epsilon_{p}=+$ for $p=2,5,7$ and $\epsilon_{p}=-$ for $p=3, \vartheta_{\gamma}$ is the theta function of $\gamma+N$ and $F_{\gamma}$ the component corresponding to $\gamma+N$ of the lift of $1 / \eta(\tau)^{m} \eta(p \tau)^{m}$ to the lattice $N$.

Suppose the vertex operator algebra $V$ of central charge 24, trivial fusion algebra and spin-1 algebra $\hat{A}_{q, p}^{r}$ exists and admits a real form. Then the cohomology group of ghost number 1 of the BRST-operator $Q$ acting on the vertex superalgebra

$$
V \otimes V_{I I_{1,1}} \otimes V_{b, c}
$$

gives a natural realization of the generalized Kac-Moody algebra corresponding to the elements of order $p$ in $M_{23}$.

For $p=2$ these results are also obtained in [13]. The advantage of the methods used here is that they do not use explicit formulas for the string functions which are in general unknown so that they can probably be applied to all the prospective vertex operator algebras in [21]. 
The above result gives evidence to the conjecture that the generalized Kac-Moody algebras whose denominator identities are completely reflective automorphic products of singular weight on lattices of squarefree level describe bosonic strings moving on suitable spacetimes.

The paper is organized as follows.

In Section 2, we recall some results on the affine Kac-Moody algebras and their highest weight representations.

In Section 3, we describe the Weil representation of $\mathrm{SL}_{2}(\mathbb{Z})$ and construct vector valued modular forms.

In Section 4, we recall some properties of vertex operator algebras and Wess-Zumino-Witten (WZW) models.

In Section 5, we show that the character of the prospective vertex operator algebra $V$ in [21] of spin-1 algebra $\hat{A}_{q, p}^{r}$ can be written in the form $\chi=\sum F_{\gamma} \vartheta_{\gamma}$ as described above.

In the last section we show that the physical states of a chiral bosonic string with vertex algebra $V \otimes V_{I I_{1,1}}$ give a realization of the generalized Kac-Moody algebra corresponding to the elements of order $p$ in $M_{23}$.

\section{Affine Kac-Moody algebras}

In this section, we recall some results on the untwisted affine Kac-Moody algebras and their highest weight representations from [14].

Let $g$ be a finite-dimensional simple complex Lie algebra with Cartan subalgebra $h$ and root system $\Delta$. Then $g$ has an invariant symmetric bilinear form which is nondegenerate on $g$ and on $h$, so that there is a natural isomorphism $\nu: h \rightarrow h^{*}$. We will often write $\alpha^{2}$ for $(\alpha, \alpha)$. The coroot $\alpha^{\vee}$ of a root $\alpha$ is the inverse image of $2 \alpha / \alpha^{2}$ under $\nu$. Let $\left\{\alpha_{1}, \ldots, \alpha_{l}\right\}$ be a set of simple roots of $g$ and $a_{i j}=\alpha_{j}\left(\alpha_{i}^{\vee}\right)$ the corresponding Cartan matrix. We denote the set of positive roots by $\Delta_{+}$. The reflections in the hyperplanes orthogonal to the simple roots generate the Weyl group $W$. The Lie algebra $g$ has at most two different root lengths. The highest root

$$
\theta=\sum_{i=1}^{l} a_{i} \alpha_{i}
$$

is a long root and

$$
\theta^{\vee}=\sum_{i=1}^{l} a_{i}^{\vee} \alpha_{i}^{\vee} .
$$

We normalize the bilinear form such that $\theta^{2}=2$. 
The untwisted affine Kac-Moody algebra corresponding to $g$ is

$$
\hat{g}=\mathbb{C}\left[t, t^{-1}\right] \otimes g \oplus \mathbb{C} K \oplus \mathbb{C} d
$$

where $K$ is central and

$$
\begin{aligned}
{\left[t^{m} \otimes x, t^{n} \otimes y\right] } & =t^{m+n} \otimes[x, y]+m \delta_{m+n}(x, y) K \\
{\left[d, t^{n} \otimes y\right] } & =n t^{n} \otimes y .
\end{aligned}
$$

The vector space

$$
\hat{h}=h \oplus \mathbb{C} K \oplus \mathbb{C} d
$$

is a commutative subalgebra of $\hat{g}$. We extend a linear function $\lambda$ on $h$ to $\hat{h}$ by setting $\lambda(K)=\lambda(d)=0$. We define linear functions $\Lambda_{0}$ and $\delta$ on $\hat{h}$ by

$$
\begin{aligned}
\Lambda_{0}(h \oplus \mathbb{C} d)=0, & \Lambda_{0}(K)=1, \\
\delta(h \oplus \mathbb{C} K)=0, & \delta(d)=1 .
\end{aligned}
$$

Then

$$
\hat{h}^{*}=h^{*} \oplus \mathbb{C} \Lambda_{0} \oplus \mathbb{C} \delta
$$

and we have a natural projection $\hat{h}^{*} \rightarrow h^{*}, \lambda \mapsto \bar{\lambda}$ with $\overline{\Lambda_{0}}=\bar{\delta}=0$. A linear function $\lambda$ in $\hat{h}^{*}$ can be written

$$
\lambda=\bar{\lambda}+\lambda(K) \Lambda_{0}+\lambda(d) \delta
$$

and $\lambda(K)$ is called the level of $\lambda$. We also extend the bilinear form from $g$ to $\hat{g}$ by setting

$$
\begin{gathered}
\left(t^{m} \otimes x, t^{n} \otimes y\right)=\delta_{m+n}(x, y) \\
\left(t^{m} \otimes x, K\right)=\left(t^{m} \otimes x, d\right)=0 \\
(K, K)=(d, d)=0 \\
(K, d)=1 .
\end{gathered}
$$

Define

$$
\alpha_{0}=\delta-\theta \text {. }
$$

Then

$$
\alpha_{0}^{\vee}=K-\theta^{\vee}
$$


and $\left\{\alpha_{0}, \alpha_{1}, \ldots, \alpha_{l}\right\}$ is a set of simple roots of $\hat{g}$ and $\left\{\alpha_{0}^{\vee}, \alpha_{1}^{\vee}, \ldots, \alpha_{l}^{\vee}\right\}$ is the set of coroots. The fundamental weights $\Lambda_{0}, \ldots, \Lambda_{l}$ satisfy

$$
\Lambda_{i}\left(\alpha_{j}^{\vee}\right)=\delta_{i j}, \quad \Lambda_{i}(d)=0
$$

Then $\Lambda_{i}=\overline{\Lambda_{i}}+a_{i}^{\vee} \Lambda_{0}$ and $\overline{\Lambda_{1}}, \ldots, \overline{\Lambda_{l}}$ are the fundamental weights of $g$. Let $b_{i j}$ be the inverse of the Cartan matrix of $g$. The scalar products of the fundamental weights are

$$
\left(\Lambda_{0}, \Lambda_{i}\right)=0
$$

for $i=0, \ldots, l$ and

$$
\left(\Lambda_{i}, \Lambda_{j}\right)=\left(\overline{\Lambda_{i}}, \overline{\Lambda_{j}}\right)=\frac{a_{i}^{\vee}}{a_{i}} b_{i j}
$$

for $i, j=1, \ldots, l$.

The Weyl vector $\rho \in \hat{h}^{*}$ is defined by $\rho\left(\alpha_{i}^{\vee}\right)=1$ for $i=0, \ldots, l$ and $\rho(d)=0$ ([14], p. 82). The level of the Weyl vector is the dual Coxeter number $h^{\vee}$.

We can write $\Lambda \in \hat{h}^{*}$ as

$$
\Lambda=\sum_{i=0}^{l} n_{i} \Lambda_{i}+c \delta
$$

with labels $n_{i}=\Lambda\left(\alpha_{i}^{\vee}\right)$ and $c=\Lambda(d)$. In representation theory, the value of $c$ is often unimportant. For example, if two weights $\Lambda$ and $\Lambda^{\prime}$ have the same labels, the corresponding irreducible highest weight modules $L(\Lambda)$ and $L\left(\Lambda^{\prime}\right)$ are isomorphic as irreducible modules of the derived algebra $\hat{g}^{\prime}=[\hat{g}, \hat{g}]$.

Let

$$
P=\left\{\lambda \in \hat{h}^{*} \mid \lambda\left(\alpha_{i}^{\vee}\right) \in \mathbb{Z} \text { for } i=0, \ldots, l\right\}
$$

be the weight lattice and

$$
P_{+}=\left\{\lambda \in P \mid \lambda\left(\alpha_{i}^{\vee}\right) \geq 0 \text { for } i=0, \ldots, l\right\}
$$

be the set of dominant integral weights. Then

$$
P=\sum_{i=0}^{l} \mathbb{Z} \Lambda_{i}+\mathbb{C} \delta .
$$

We define $P^{k}=\{\lambda \in P \mid \lambda(K)=k\}$ and $P_{+}^{k}=P^{k} \cap P_{+}$. 
Let $\Lambda \in P_{+}^{k}$ with $k>0$ and $L(\Lambda)$ the corresponding irreducible highest weight module. We define

$$
m_{\Lambda}=\frac{(\Lambda+\rho)^{2}}{2\left(k+h^{\vee}\right)}-\frac{\rho^{2}}{2 h^{\vee}}
$$

and the normalized character

$$
\chi_{\Lambda}=e^{-m_{\Lambda} \delta} \operatorname{ch} L(\Lambda) .
$$

For $\lambda \in \hat{h}^{*}$ we define

$$
m_{\Lambda, \lambda}=m_{\Lambda}-\frac{\lambda^{2}}{2 k}
$$

and the string function

$$
c_{\lambda}^{\Lambda}=e^{-m_{\Lambda, \lambda} \delta} \sum_{n \in \mathbb{Z}} \operatorname{mult}_{L(\Lambda)}(\lambda-n \delta) e^{-n \delta} .
$$

The string functions are invariant under the action of the affine Weyl group, i.e.,

$$
c_{w(\lambda)}^{\Lambda}=c_{\lambda}^{\Lambda}
$$

for $w \in \hat{W}$, so that

$$
c_{w(\lambda)+k \gamma+a \delta}^{\Lambda}=c_{\lambda}^{\Lambda}
$$

for $w \in W, \gamma \in M$ and $a \in \mathbb{C}([14],(12.7 .9))$.

The multiplicities can be calculated with Freudenthal's recursion formula

$$
\begin{aligned}
& \left((\Lambda+\rho)^{2}-(\lambda+\rho)^{2}\right) \operatorname{mult}_{L(\Lambda)}(\lambda) \\
& \quad=2 \sum_{\alpha \in \Delta_{+}} \sum_{j \geq 1} \operatorname{mult}_{L(\Lambda)}(\alpha)(\lambda+j \alpha, \alpha) \operatorname{mult}_{L(\Lambda)}(\lambda+j \alpha) .
\end{aligned}
$$

Let

$$
\theta_{\lambda}=e^{k \Lambda_{0}} \sum_{\gamma \in M+\bar{\lambda} / k} e^{-k \gamma^{2} \delta / 2+k \gamma}
$$

where $M$ is the lattice generated by the long roots of $g([14],(12.7 .3))$. Then

$$
\theta_{\lambda+k \gamma+a \delta}=\theta_{\lambda}
$$

for $\gamma \in M$ and $a \in \mathbb{C}([14],(13.2 .3))$. 
The normalized character is given by

$$
\chi_{\Lambda}=\sum_{\lambda \in P^{k} \bmod (k M+\mathbb{C} \delta)} c_{\lambda}^{\Lambda} \theta_{\lambda}
$$

$([14],(12.7 .12))$.

We obtain modular forms if we replace in the above definitions $e^{-\delta}=q$ with $q=e^{2 \pi i \tau}$. The string functions transform under the generators of $\mathrm{SL}_{2}(\mathbb{Z})$ as

$$
c_{\lambda}^{\Lambda}(-1 / \tau)=\left|M^{\prime} / k M\right|^{-1 / 2}(-i \tau)^{-l / 2} \sum_{\substack{\Lambda^{\prime} \in P_{+}^{k} \bmod \mathbb{C} \delta \\ \lambda^{\prime} \in P^{k} \bmod (k M+\mathbb{C} \delta)}} S_{\Lambda, \Lambda^{\prime}} e\left(\frac{\left(\bar{\lambda}, \overline{\lambda^{\prime}}\right)}{k}\right) c_{\lambda^{\prime}}^{\Lambda^{\prime}}(\tau)
$$

with

$$
S_{\Lambda, \Lambda^{\prime}}=i^{\left|\Delta_{+}\right|}\left|M^{\prime} /\left(k+h^{\vee}\right) M\right|^{-1 / 2} \sum_{w \in W} \operatorname{det}(w) e\left(\frac{-\left(\bar{\Lambda}+\bar{\rho}, w\left(\overline{\Lambda^{\prime}}+\bar{\rho}\right)\right)}{\left(k+h^{\vee}\right)}\right)
$$

([14], Theorems 13.8 and 13.10) and

$$
c_{\lambda}^{\Lambda}(\tau+1)=e\left(m_{\Lambda, \lambda}\right) c_{\lambda}^{\Lambda}(\tau)
$$

$([14],(13.10 .4))$.

\section{The Weil representation}

In this section, we describe the Weil representation of $\mathrm{SL}_{2}(\mathbb{Z})$ and construct vector valued modular forms. More details can be found in [20].

A discriminant form is a finite abelian group $D$ with a quadratic form $D \rightarrow \mathbb{Q} / \mathbb{Z}, \gamma \mapsto \gamma^{2} / 2$ such that $(\beta, \gamma)=(\beta+\gamma)^{2} / 2-\beta^{2} / 2-\gamma^{2} / 2 \bmod 1$ is a nondegenerate symmetric bilinear form. The level of $D$ is the smallest positive integer $N$ such that $N \gamma^{2} / 2 \in \mathbb{Z}$ for all $\gamma \in D$. The group algebra $\mathbb{C}[D]$ of $D$ is the algebra with basis $\left\{e^{\gamma} \mid \gamma \in D\right\}$ and products $e^{\beta} e^{\gamma}=e^{\beta+\gamma}$.

Let $L$ be an even lattice with dual lattice $L^{\prime}$. Then $L^{\prime} / L$ is a discriminant form with the quadratic form given by $\gamma^{2} / 2 \bmod 1$. Conversely, every discriminant form can be obtained in this way. The signature $\operatorname{sign}(D) \in \mathbb{Z} / 8 \mathbb{Z}$ of a discriminant form is defined as the signature modulo 8 of any even lattice with that discriminant form. 
Let $D$ be a discriminant form of even signature. There is a unitary action of the group $\mathrm{SL}_{2}(\mathbb{Z})$ on $\mathbb{C}[D]$ defined by

$$
\begin{aligned}
& \rho_{D}(T) e^{\gamma}=e\left(-\gamma^{2} / 2\right) e^{\gamma} \\
& \rho_{D}(S) e^{\gamma}=\frac{e(\operatorname{sign}(D) / 8)}{\sqrt{|D|}} \sum_{\beta \in D} e((\gamma, \beta)) e^{\beta},
\end{aligned}
$$

where $S=\left(\begin{array}{cc}0 & -1 \\ 1 & 0\end{array}\right)$ and $T=\left(\begin{array}{ll}1 & 1 \\ 0 & 1\end{array}\right)$ are the standard generators of $\mathrm{SL}_{2}(\mathbb{Z})$. This representation is called Weil representation.

Let

$$
F(\tau)=\sum_{\gamma \in D} F_{\gamma}(\tau) e^{\gamma}
$$

be a holomorphic function on the upper halfplane with values in $\mathbb{C}[D]$ and $k$ an integer. Then $F$ is a modular form for $\rho_{D}$ of weight $k$ if

$$
F(M \tau)=(c \tau+d)^{k} \rho_{D}(M) F(\tau)
$$

for all $M=\left(\begin{array}{ll}a & b \\ c & d\end{array}\right)$ in $\mathrm{SL}_{2}(\mathbb{Z})$ and $F$ is meromorphic at $\infty$.

We can construct modular forms for the Weil representation by lifting scalar valued modular forms on $\Gamma_{0}(N)$. Suppose that the level of $D$ divides $N$, where $N$ is a positive integer. Let $f$ be a scalar valued modular form on $\Gamma_{0}(N)$ of weight $k$ and character $\chi_{D}$. Again we allow poles at cusps. Then

$$
F(\tau)=\left.\sum_{M \in \Gamma_{0}(N) \backslash \Gamma} f\right|_{M}(\tau) \rho_{D}\left(M^{-1}\right) e^{0}
$$

is a vector valued modular form for $\rho_{D}$ of weight $k$ which is invariant under the automorphisms of the discriminant form.

Now we consider the following cases. Let $p=2,3,5$ or 7 . Then there is an automorphism of the Leech lattice of cycle shape $1^{m} p^{m}$ with $m=24 /(p+1)$. The fixpoint lattice $\Lambda_{p}$ is the unique lattice in its genus without roots. Let $I I_{1,1}$ be the even unimodular Lorentzian lattice of rank 2 . Then the lattice

$$
\Lambda_{p} \oplus \sqrt{p} I_{1,1}
$$

has level $p$ and genus

$$
I I_{2 m+1,1}\left(p^{\epsilon_{p}(m+2)}\right)
$$

with $\epsilon_{p}=+,-,+,+$ for $p=2,3,5,7$. The eta product

$$
f(\tau)=\frac{1}{\eta(\tau)^{m} \eta(p \tau)^{m}}
$$


is a modular form for $\Gamma_{0}(p)$ of weight $-m$ with poles at the cusps 0 and $\infty$ and trivial character for $p=2,3$ and 5 and character $\chi\left(\left(\begin{array}{ll}a & b \\ c & d\end{array}\right)\right)=\left(\frac{d}{7}\right)$ in the case $p=7$. We define $T$-invariant functions $g_{j}$ by

$$
f\left(\frac{\tau}{p}\right)=g_{0}(\tau)+g_{1}(\tau)+\cdots+g_{p-1}(\tau)
$$

with $\left.g_{j}\right|_{T}(\tau)=e(j / p) g_{j}(\tau)$, i.e.,

$$
g_{j}(\tau)=\frac{1}{p} \sum_{k=0}^{p-1} e\left(-\frac{k j}{p}\right) f\left(\frac{\tau+k}{p}\right) .
$$

We lift the modular form $f$ to a modular form $F=\sum F_{\gamma} e^{\gamma}$ on $\Lambda_{p} \oplus \sqrt{p} I I_{1,1}$ using the above construction. Then $F$ has components

$$
F_{\gamma}(\tau)= \begin{cases}f(\tau)+g_{0}(\tau) & \text { if } \gamma=0 \\ g_{j}(\tau) & \text { if } \gamma \neq 0 \text { and } \gamma^{2} / 2=-j / p \bmod 1\end{cases}
$$

The components $F_{\gamma}$ with $\gamma^{2} / 2=0 \bmod 1$ are modular forms for $\Gamma_{0}(p)$ of weight $-m$ and of nontrivial quadratic character in the case $p=7$.

\section{WZW theories}

In this section, we recall some results on vertex operator algebras and WZW models. References are [7, 9-11,22].

Let $V$ be a vertex operator algebra satisfying certain regularity properties. In particular, we assume that $V$ is simple and rational, i.e., $V$ is irreducible as a module over itself and $V$ has only finitely many nonisomorphic simple modules and every module decomposes into a finite direct sum of irreducible modules. Let $\left\{M_{0}, \ldots, M_{n}\right\}$ with $M_{0}=V$ be the set of nonisomorphic simple modules. The dimensions of the intertwining spaces

$$
N_{i j}^{k}=\operatorname{dim}\left(\begin{array}{c}
M_{k} \\
M_{i} M_{j}
\end{array}\right)
$$

satisfy $N_{i j}^{k}=N_{j i}^{k}, N_{0 i}^{j}=\delta_{i}^{j}$ and $N_{i j}^{0}=\delta_{j i^{+}}$for a unique $i^{+}$depending on $i$. The fusion algebra is the free vector space $\mathbb{C}[I]$, where $I=\{0, \ldots, n\}$ with products

$$
i \times j=\sum_{k \in I} N_{i j}^{k} k
$$

The fusion algebra is commutative, associative and $i \times 0=i$. 
Zhu [22] has shown that the space of genus 1 correlation functions of $V$ is invariant under $\mathrm{SL}_{2}(\mathbb{Z})$ and has a basis corresponding to the modules $M_{i}$. We denote the $S$-matrix with respect to this basis by $S_{i j}$. A consequence of this result is that the characters $\chi_{i}$ of the modules $M_{i}$ are invariant under $\mathrm{SL}_{2}(\mathbb{Z})$.

The $S$-matrix is related to the structure constants of the fusion algebra by the Verlinde formula (cf. [12])

$$
N_{i j}^{k}=\sum_{n \in I} \frac{S_{i n} S_{j n} S_{k n}^{-1}}{S_{0 n}} .
$$

A module $M_{i}$ is called a simple current if for each $j$ there is a unique $k$ such that $i \times j=k$. Then of course $i \times i^{+}=0$. This condition is also sufficient, i.e., $M_{i}$ is a simple current if and only if $N_{i i^{+}}^{j}=\delta^{j 0}$. A simple current generates an action of a group $\mathbb{Z} / N \mathbb{Z}$ on the set of modules.

An important problem is when there is a vertex algebra structure on a sum over the modules $M_{i}$ extending $V$. In some special cases, e.g., simple current extensions of WZW models, this problem has been solved.

Let $g$ be a finite-dimensional simple complex Lie algebra with affinization $\hat{g}$ and $k$ a positive integer. Then the irreducible $\hat{g}$-module $L\left(k \Lambda_{0}\right)$ has a canonical structure as a simple rational vertex operator algebra which satisfies the above mentioned regularity properties. The simple modules are the irreducible $\hat{g}$-modules $L(\Lambda)$ with $\Lambda \in P_{+}^{k}$ and $\Lambda(d)=0$. This theory is called the WZW model corresponding to $g$ of level $k$. Since in this case, the $S$-matrix is known, the structure constants of the fusion algebra can be calculated by the Verlinde formula. Fuchs [8] has determined the simple currents of the WZW models. The nontrivial simple currents correspond to the weights $\Lambda$, where $\bar{\Lambda}$ is $k$ times a cominimal weight of $g$. If $g$ is $E_{8}$ there is an exceptional simple current at level 2 .

Let $V$ be a vertex operator algebra. Then the $L_{0}$-eigenspace $V_{1}$ is a Lie algebra under $[u, v]=u_{0} v$ with invariant bilinear form $(u, v)=u_{1} v$. The components of the fields $u(z)=\sum_{m \in \mathbb{Z}} u_{m} z^{-m-1}$ and $v(z)=\sum_{n \in \mathbb{Z}} v_{n} z^{-n-1}$ satisfy the commutation relations

$$
\left[u_{m}, v_{n}\right]=([u, v])_{m+n}+m(u, v) \delta_{m+n} 1 .
$$

Schellekens [21] studies the character valued partition functions of meromorphic vertex operator algebras of central charge 24. With the help of the level 1 trace identities he proves the following result. Let $V$ be a vertex operator algebra of central charge 24 , trivial fusion algebra and nonzero $V_{1}$. 
Then either $\operatorname{dim} V_{1}=24$ and $V_{1}$ is commutative or $\operatorname{dim} V_{1}>24$ and $V_{1}$ is semisimple. In the first case, $V$ is the vertex operator algebra of the Leech lattice. In the second case, $V$ can be written as a sum of modules over the affinization $\hat{V}_{1}$ of $V_{1}$. Using the trace identities of level 2, he shows that there are at most 69 possibilities for the Lie algebra $V_{1}$ if $\operatorname{dim} V_{1}>24$. For each of these possibilities, he finds exactly one modular invariant partition function and describes explicitly the decomposition of $V$ as $\hat{V}_{1}$-module. For many of these vector spaces, it is still open whether they have a vertex algebra structure because the extension problem for WZW models has not been solved in general so far. Schellekens' results suggest that there are 71 meromorphic vertex operator algebras of central charge 24 , the monster vertex operator algebra, the vertex operator algebra of the Leech lattice and the 69 vertex operator algebras with Kac-Moody symmetry.

\section{Characters of some vertex operator algebras}

In this section, we show that the character of the prospective conformal field theory in [21] of spin-1 algebra $\hat{A}_{p-1, p}^{r}$ can be written in the form $\chi=$ $\sum F_{\gamma} \vartheta_{\gamma}$.

Let $p=2,3,5$ or 7 and $q=p-1$.

First, we describe some properties of the affine Kac-Moody algebra $\hat{A}_{q}$. The central element $K$ is given by

$$
K=\alpha_{0}^{\vee}+\cdots+\alpha_{q}^{\vee}
$$

Let

$$
\lambda=n_{0} \Lambda_{0}+\cdots+n_{q} \Lambda_{q}=\left(n_{0}, \ldots, n_{q}\right)
$$

be in $P \bmod \mathbb{C} \delta$. Then $\lambda$ has level

$$
\lambda(K)=n_{0}+\cdots+n_{q}
$$

and norm

$$
(\lambda, \lambda)=\lambda^{2}=\sum_{i, j=1}^{q} b_{i j} n_{i} n_{j},
$$

where $b_{i j}$ is the inverse of the Cartan matrix of $A_{q}$. 
The natural projection from $P \bmod \mathbb{C} \delta$ to the lattice $A_{q}^{\prime}$ sends

$$
\lambda=n_{0} \Lambda_{0}+\cdots+n_{q} \Lambda_{q}
$$

to

$$
\bar{\lambda}=n_{1} \overline{\Lambda_{1}}+\cdots+n_{q} \overline{\Lambda_{q}}
$$

This map induces a bijection from the weights of level $p$ in $P \bmod \mathbb{C} \delta$ to the lattice $A_{q}^{\prime}$.

The group $A_{q}^{\prime} / A_{q} \cong \mathbb{Z} / p \mathbb{Z}$ is sometimes called congruence group and its elements congruence classes. It can be represented by the elements $\overline{\Lambda_{i}}$ with $\overline{\Lambda_{i}}+\overline{\Lambda_{j}}=\overline{\Lambda_{i+j}}$ where addition is taken modulo $p$.

Let $\lambda=p \Lambda_{0}=(p, 0, \ldots, 0)$. Then the irreducible highest weight module $L(\lambda)$ is a vertex operator algebra whose irreducible modules correspond to the weights $\left(n_{0}, \ldots, n_{q}\right)$, where the $n_{i}$ are nonnegative integers and $n_{0}+$ $\cdots+n_{q}=p$. The simple currents are given by the weights $p \Lambda_{i}$. They act by cyclicly shifting the coefficients to the right, i.e., $\left(p \Lambda_{i}\right) \cdot\left(n_{0}, \ldots, n_{q}\right)=$ $\left(n_{q+1-i}, \ldots, n_{q-i}\right)$, and form a group isomorphic to $\mathbb{Z} / p \mathbb{Z}$.

We describe some properties of the simple currents.

Let $\lambda$ be a weight of level $p$ and $s$ a simple current. Then $\bar{\lambda}$ and $\overline{s . \lambda}$ are in the same class.

Furthermore

Proposition 5.1. The string functions of $\hat{A}_{q}$ of level $p$ are invariant under the following action of a simple current $s$

$$
c_{\lambda}^{\Lambda}=c_{s . \lambda}^{s . \Lambda}
$$

Proof. There is a diagram automorphism $\phi$ acting on $\bigoplus_{i=0}^{l} \mathbb{C} \alpha_{i}$ such that $\phi . \alpha_{i} \bmod \mathbb{C} \delta=s .\left(\alpha_{i} \bmod \mathbb{C} \delta\right)$ for $i=0, \ldots, l$. We extend $\phi$ to $\hat{h}^{*}$ by $\phi . \Lambda_{0}=s . \Lambda_{0}$. Then there is a unique map $\phi$ on $\hat{h}$ satisfying $(\phi . \lambda)(\phi . y)=\lambda(y)$ for all $\lambda \in \hat{h}^{*}$ and $y \in \hat{h}$. This map gives an isomorphic realization and therefore an isomorphism of $\hat{g}$. Hence

$$
c_{s . \lambda}^{s . \Lambda}=c_{\phi . \lambda+c \delta}^{\phi . \Lambda+C \delta}=c_{\phi . \lambda}^{\phi . \Lambda}=c_{\lambda}^{\Lambda} .
$$

The isomorphism from the discriminant group to the group of simple currents sending $\overline{\Lambda_{i}}$ to $p \Lambda_{i}$ satisfies 
Proposition 5.2. Let $\mu$ be a weight of level 0 such that $\bar{\mu}=\overline{\Lambda_{i}} \bmod A_{q}$. Then

for all $\Lambda$ in $P_{+}^{p}$ and all $\lambda$ in $P^{p}$.

$$
c_{\lambda+p \mu}^{\Lambda}=c_{\left(p \Lambda_{i}\right) . \lambda}^{\Lambda}
$$

Proof. Let $\lambda=\left(n_{0}, \ldots, n_{q}\right)$ be a weight of level $p$ and $\mu=\left(m_{0}, \ldots, m_{q}\right)$ a weight of level 0 such that $\bar{\mu}=\overline{\Lambda_{1}} \bmod A_{q}$. Then

$$
\begin{aligned}
w_{1} w_{2} \ldots w_{q}(\lambda+p \mu) & =\left(n_{q}, n_{0}, \ldots, n_{q-1}\right) \bmod A_{q} \\
& =\left(p \Lambda_{1}\right) \cdot \lambda \bmod A_{q}
\end{aligned}
$$

so that

$$
c_{\lambda+p \mu}^{\Lambda}=c_{w_{1} w_{2} \ldots w_{q}(\lambda+p \mu)}^{\Lambda}=c_{\left(p \Lambda_{1}\right) \cdot \lambda}^{\Lambda} .
$$

This implies the statement.

Let $V$ be the prospective vertex operator algebra in [21] of spin-1 algebra $\hat{A}_{q, p}^{r}$, where $r=48 / q(p+1)$. Then $V$ is a sum of irreducible highest weight modules of $\hat{A}_{q}^{r}$, the weight of each factor $\hat{A}_{q}$ having level $p$. We denote the set of highest weight vectors by $M$. The set $M$ is invariant under the natural action of a subgroup $G$ of the group of simple currents and decomposes into $G$-orbits $G \backslash M$. In the appendix, we list $G$ and orbit representatives of $G \backslash M$ together with their multiplicities.

Using the isomorphism from the discriminant group to the group of simple currents we can consider $G$ as subgroup of $A_{q}^{r \prime} / A_{q}^{r}$. We denote by $\left(A_{q}^{r}, G\right)$ the rational lattice obtained by gluing the elements of $G$ to $A_{q}^{r}$ and analogously by $\left(A_{q}^{r}, G^{\perp}\right)$ the rational lattice obtained by gluing the elements of the orthogonal complement $G^{\perp}$ to $A_{q}^{r}$. Note that $\left(A_{q}^{r}, G^{\perp}\right)$ is the dual lattice of $\left(A_{q}^{r}, G\right)$.

The character of $V$ as $\hat{A}_{q}^{r}$-module is given by

$$
\begin{aligned}
\chi_{V} & =\sum_{\Lambda \in M} \operatorname{mult}(\Lambda) \chi_{\Lambda} \\
& =\sum_{\Lambda \in M} \operatorname{mult}(\Lambda) \prod_{i=1}^{r} \sum_{\lambda_{i} \in A_{q}^{\prime} / p A_{q}} c_{\lambda_{i}}^{\Lambda_{i}}(\tau) \theta_{\lambda_{i}}\left(\tau, z_{i}, u_{i}\right) \\
& =\sum_{\lambda \in A_{q}^{r^{\prime}} / p A_{q}^{r}} \sum_{\Lambda \in M} \operatorname{mult}(\Lambda) \prod_{i=1}^{r} c_{\lambda_{i}}^{\Lambda_{i}}(\tau) \theta_{\lambda_{i}}\left(\tau, z_{i}, u_{i}\right) .
\end{aligned}
$$


We have

Proposition 5.3. The only nonzero contributions in the above expression for the character of $V$ come from weights $\lambda$ in $\left(A_{q}^{r}, G^{\perp}\right)$.

Proof. A case-by-case analysis shows $M \subset\left(A_{q}^{r}, G^{\perp}\right)$. It is easy to see that $c_{\lambda}^{\Lambda}=0$ if $\Lambda$ and $\lambda$ are not in the same class. Therefore any nonzero contribution to the character of $V$ comes from a weight $\lambda$ in $\left(A_{q}^{r}, G^{\perp}\right)$.

It follows

Proposition 5.4. The character of $V$ as $\hat{A}_{q}^{r}$-module can be written as

$$
\chi_{V}=\sum_{\lambda \in N^{\prime} / N} \tilde{F}_{\lambda} \vartheta_{\lambda}
$$

where $N=\sqrt{p}\left(A_{q}^{r}, G\right), \vartheta_{\lambda}$ is the theta function of the coset $\lambda+N$ and

$$
\tilde{F}_{\lambda}=\sum_{\Lambda \in G \backslash M} \sum_{g \in G} \frac{\operatorname{mult}(\Lambda)}{\left|G_{\Lambda}\right|} \prod_{i=1}^{r} c_{g_{i} \cdot \sqrt{p} \lambda_{i}}^{\Lambda_{i}}
$$

Proof. By Propositions 5.3 and 5.1 we have

$$
\begin{aligned}
\chi_{V} & =\sum_{\lambda \in A_{q}^{r^{\prime}} / p A_{q}^{r}} \sum_{\Lambda \in M} \operatorname{mult}(\Lambda) \prod_{i=1}^{r} c_{\lambda_{i}}^{\Lambda_{i}}(\tau) \theta_{\lambda_{i}}\left(\tau, z_{i}, u_{i}\right) \\
& =\sum_{\lambda \in\left(A_{q}^{r}, G^{\perp}\right) / p A_{q}^{r}} \sum_{\Lambda \in M} \operatorname{mult}(\Lambda) \prod_{i=1}^{r} c_{\lambda_{i}}^{\Lambda_{i}}(\tau) \theta_{\lambda_{i}}\left(\tau, z_{i}, u_{i}\right) \\
& =\sum_{\lambda \in\left(A_{q}^{r}, G^{\perp}\right) / p A_{q}^{r}} \sum_{\Gamma \in G \backslash M} \sum_{\Lambda \in G . \Gamma} \operatorname{mult}(\Gamma) \prod_{i=1}^{r} c_{\lambda_{i}}^{\Lambda_{i}}(\tau) \theta_{\lambda_{i}}\left(\tau, z_{i}, u_{i}\right) \\
& =\sum_{\lambda \in\left(A_{q}^{r}, G^{\perp}\right) / p A_{q}^{r}} \sum_{\Lambda \in G \backslash M} \sum_{g \in G} \frac{\operatorname{mult}(\Lambda)}{\left|G_{\Lambda}\right|} \prod_{i=1}^{r} c_{\lambda_{i}}^{g_{i} . \Lambda_{i}}(\tau) \theta_{\lambda_{i}}\left(\tau, z_{i}, u_{i}\right) \\
& =\sum_{\lambda \in\left(A_{q}^{r}, G^{\perp}\right) / p A_{q}^{r}} \sum_{\Lambda \in G \backslash M}^{r} \sum_{g \in G} \frac{\operatorname{mult}(\Lambda)}{\left|G_{\Lambda}\right|} \prod_{i=1}^{r} c_{g_{i} \cdot \lambda_{i}}^{\Lambda_{i}}(\tau) \theta_{\lambda_{i}}\left(\tau, z_{i}, u_{i}\right) \\
& =\sum_{\lambda \in\left(A_{q}^{r}, G^{\perp}\right) / p A_{q}^{r}} \prod_{i=1} \theta_{\lambda_{i}}\left(\tau, z_{i}, u_{i}\right) \sum_{\Lambda \in G \backslash M} \sum_{g \in G} \frac{\operatorname{mult}(\Lambda)}{\left|G_{\Lambda}\right|} \prod_{i=1}^{r} c_{g_{i} . \lambda_{i}}^{\Lambda_{i}}(\tau),
\end{aligned}
$$


where $G_{\Lambda}$ denotes the stabilizer of $\Lambda$ in $G$. Using Proposition 5.2 we get

$$
\begin{aligned}
\chi_{V}= & \sum_{\lambda \in\left(A_{q}^{r}, G^{\perp}\right) / p\left(A_{q}^{r}, G\right)} \sum_{\mu \in p\left(A_{q}^{r}, G\right) / p A_{q}^{r}} \prod_{i=1}^{r} \theta_{\lambda_{i}+\mu_{i}}\left(\tau, z_{i}, u_{i}\right) \\
& \cdot \sum_{\Lambda \in G \backslash M} \sum_{g \in G} \frac{\operatorname{mult}(\Lambda)}{\left|G_{\Lambda}\right|} \prod_{i=1}^{r} c_{g_{i} \cdot\left(\lambda_{i}+\mu_{i}\right)}^{\Lambda_{i}}(\tau) \\
= & \sum_{\lambda \in\left(A_{q}^{r}, G^{\perp}\right) / p\left(A_{q}^{r}, G\right)} \sum_{\mu \in p\left(A_{q}^{r}, G\right) / p A_{q}^{r}} \prod_{i=1}^{r} \theta_{\lambda_{i}+\mu_{i}}\left(\tau, z_{i}, u_{i}\right) \\
& \cdot \sum_{\Lambda \in G \backslash M} \sum_{g \in G} \frac{\operatorname{mult}(\Lambda)}{\left|G_{\Lambda}\right|} \prod_{i=1}^{r} c_{s_{i} \cdot g_{i} \cdot \lambda_{i}}^{\Lambda_{i}}(\tau) \\
= & \sum_{\lambda \in\left(A_{q}^{r}, G^{\perp}\right) / p\left(A_{q}^{r}, G\right) \mu \in p\left(A_{q}^{r}, G\right) / p A_{q}^{r}} \prod_{i=1}^{r} \theta_{\lambda_{i}+\mu_{i}}\left(\tau, z_{i}, u_{i}\right) \\
& \cdot \sum_{\Lambda \in G \backslash M} \sum_{g \in G} \frac{\operatorname{mult}(\Lambda)}{\left|G_{\Lambda}\right|} \prod_{i=1}^{r} c_{g_{i} \cdot \lambda_{i}}^{\Lambda_{i}}(\tau) .
\end{aligned}
$$

Here $s_{i}$ is the simple current of $\hat{A}_{q}$ such that $c_{g_{i} . \lambda_{i}+g_{i} . \mu_{i}}^{\Lambda_{i}}=c_{s_{i} \cdot g_{i}, \lambda_{i}}^{\Lambda_{i}}$. Note that $s=\left(s_{1}, \ldots, s_{r}\right)$ is in $G$ because $\mu=\left(\mu_{1}, \ldots, \mu_{r}\right)$ is in $p\left(A_{q}^{r}, G\right) / p A_{q}^{r}$. Now

$$
\begin{aligned}
& \sum_{\mu \in p\left(A_{q}^{r}, G\right) / p A_{q}^{r}} \prod_{i=1}^{r} \theta_{\lambda_{i}+\mu_{i}}\left(\tau, z_{i}, u_{i}\right) \\
= & \sum_{\mu \in p\left(A_{q}^{r}, G\right) / p A_{q}^{r}} \sum_{\nu \in p A_{q}^{r}} \prod_{i=1}^{r} e\left(p u_{i}\right) e\left(\frac{\tau\left(\lambda_{i}+\mu_{i}+\nu_{i}\right)^{2}}{2 p}+\left(\lambda_{i}+\mu_{i}+\nu_{i}, z_{i}\right)\right) \\
= & \sum_{\mu \in p\left(A_{q}^{r}, G\right)} \prod_{i=1}^{r} e\left(p u_{i}\right) e\left(\frac{\tau\left(\lambda_{i}+\mu_{i}\right)^{2}}{2 p}+\left(\lambda_{i}+\mu_{i}, z_{i}\right)\right) \\
= & \sum_{\mu \in \sqrt{p}\left(A_{q}^{r}, G\right)} \prod_{i=1}^{r} e\left(p u_{i}\right) e\left(\frac{\tau\left(\lambda_{i}+\sqrt{p} \mu_{i}\right)^{2}}{2 p}+\left(\lambda_{i}+\sqrt{p} \mu_{i}, z_{i}\right)\right) \\
= & \sum_{\mu \in \sqrt{p}\left(A_{q}^{r}, G\right)} \prod_{i=1}^{r} e\left(p u_{i}\right) e\left(\frac{\tau\left(\lambda_{i} / \sqrt{p}+\mu_{i}\right)^{2}}{2}+\left(\lambda_{i} / \sqrt{p}+\mu_{i}, \sqrt{p} z_{i}\right)\right),
\end{aligned}
$$


so that

$$
\begin{aligned}
\chi_{V}= & \sum_{\lambda \in\left(A_{q}^{r}, G^{\perp}\right) / p\left(A_{q}^{r}, G\right)} \sum_{\mu \in \sqrt{p}\left(A_{q}^{r}, G\right)} \prod_{i=1}^{r} e\left(p u_{i}\right) e\left(\frac{\tau\left(\lambda_{i} / \sqrt{p}+\mu_{i}\right)^{2}}{2}\right. \\
& \left.+\left(\lambda_{i} / \sqrt{p}+\mu_{i}, \sqrt{p} z_{i}\right)\right) \cdot \sum_{\Lambda \in G \backslash M} \sum_{g \in G} \frac{\operatorname{mult}(\Lambda)}{\left|G_{\Lambda}\right|} \prod_{i=1}^{r} c_{g_{i} \cdot \lambda_{i}}^{\Lambda_{i}}(\tau) \\
= & \sum_{\lambda \in(1 / \sqrt{p})\left(A_{q}^{r}, G^{\perp}\right) / \sqrt{p}\left(A_{q}^{r}, G\right)} \sum_{\mu \in \sqrt{p}\left(A_{q}^{r}, G\right)} \prod_{i=1}^{r} e\left(p u_{i}\right) e\left(\frac{\tau\left(\lambda_{i}+\mu_{i}\right)^{2}}{2}\right. \\
& \left.+\left(\lambda_{i}+\mu_{i}, \sqrt{p} z_{i}\right)\right) \cdot \sum_{\Lambda \in G \backslash M} \sum_{g \in G} \frac{\operatorname{mult}(\Lambda)}{\left|G_{\Lambda}\right|} \prod_{i=1}^{r} c_{g_{i} \cdot \sqrt{p} \lambda_{i}}^{\Lambda_{i}}(\tau) .
\end{aligned}
$$

This implies the proposition.

Note that the functions $\tilde{F}_{\gamma}$ are invariant under $G$, the permutations $\operatorname{Sym}(G)$ of the $r$ components which leave $G$ invariant and by Proposition 5.1 also under $\hat{W}^{r}$.

Proposition 5.5. The lattice $N=\sqrt{p}\left(A_{q}^{r}, G\right)$ has genus

$$
I I_{2 m, 0}\left(p^{\epsilon_{p}(m+2)}\right)
$$

where $m=24 /(p+1)$ and $\epsilon_{p}=+,-,+,+$ for $p=2,3,5,7$. In the case $p=2$, the 2-adic Jordan components are even. The minimal norm of $N$ is 4 for $p=2,3$ and 5, and 6 for $p=7$. This is the largest possible minimal norm of a lattice in this genus and $N$ is the unique lattice up to isomorphism with this minimal norm.

Proof. It is easy to see that $N$ is an even lattice. Furthermore, we have $p N^{\prime}=\sqrt{p}\left(A_{q}^{r}, G^{\perp}\right) \subset \sqrt{p}\left(A_{q}^{r}, G\right)=N$ because $G^{\perp} \subset G$. Using $|G|^{2}=p^{24 / q-2}$ we get

$$
\begin{aligned}
\operatorname{det}(N) & =\operatorname{det}\left(A_{q}^{r}, G\right)(p) \\
& =p^{r q} \operatorname{det}\left(A_{q}^{r}, G\right) \\
& =\frac{p^{r q}}{|G|^{2}} \operatorname{det}\left(A_{q}^{r}\right) \\
& =\frac{p^{r p}}{|G|^{2}} \\
& =p^{m+2}
\end{aligned}
$$


This implies that $N$ has the stated genus (cf. [6, p. $386 \mathrm{ff}$., Theorem 13]). The minimal norm of $N$ follows from the minimal distance of $G$ considered as linear code in $\mathbb{F}_{p}^{r}$. We find that the minimal norm of $N$ is 4 for $p=2,3$ and 5 , and 6 for $p=7$. We leave the proof of the other statements to the reader.

Now we determine the function

$$
\tilde{F}=\sum_{\gamma \in D} \tilde{F}_{\gamma} e^{\gamma}
$$

where $D$ is the discriminant form of $N$, explicitly.

Theorem 5.6. The function $\tilde{F}$ is a modular form of weight $-24 /(p+1)$ for the Weil representation of $N$.

Proof. We have to show that

$$
\tilde{F}_{\gamma}(\tau+1)=e\left(-\gamma^{2} / 2\right) \tilde{F}_{\gamma}(\tau)
$$

and

$$
\tilde{F}_{\gamma}\left(-\frac{1}{\tau}\right)=\frac{e(\operatorname{sign}(D) / 8)}{\sqrt{|D|}} \tau^{k} \sum_{\beta \in D} e((\gamma, \beta)) \tilde{F}_{\beta}(\tau)
$$

with $k=-24 /(p+1)$ for all $\gamma$ in $D$.

To prove these equations we proceed as follows. We choose a set of functions $\left\{\tilde{F}_{\gamma_{1}}, \tilde{F}_{\gamma_{2}}, \ldots, \tilde{F}_{\gamma_{n}}\right\}$ such that each $\tilde{F}_{\gamma}$ is conjugate to exactly one $\tilde{F}_{\gamma_{j}}$ under the action of $G, \operatorname{Sym}(G)$ and $\hat{W}^{r}$.

Using the $T$-invariance of the string functions we verify that

$$
\tilde{F}_{\gamma_{j}}(T \tau)=e\left(-\gamma_{j}^{2} / 2\right) \tilde{F}_{\gamma_{j}}(\tau) .
$$

This implies that the $\tilde{F}_{\gamma}$ transform correctly under $T$.

Let $\gamma \in D$. We define constants $c_{\gamma, \gamma_{l}}$ by

$$
\sum_{\beta \in D} e((\gamma, \beta)) \tilde{F}_{\beta}=\sum_{l} c_{\gamma, \gamma_{l}} \tilde{F}_{\gamma_{l}} .
$$

If $\gamma$ is equivalent to $\gamma_{j}$ under the above symmetries then

$$
c_{\gamma, \gamma_{l}}=c_{\gamma_{j}, \gamma_{l}}
$$


The action of $S$ on $\tilde{F}_{\gamma_{j}}$ is given by

$$
\tilde{F}_{\gamma_{j}}(S \tau)=\sum_{\Lambda \in G \backslash M} \sum_{g \in G} \frac{\operatorname{mult}(\Lambda)}{\left|G_{\Lambda}\right|} \prod_{i=1}^{r} c_{g_{i} \cdot \sqrt{p}\left(\gamma_{j}\right)_{i}}^{\Lambda_{i}}(S \tau)
$$

We determine the $S$-matrix of the string functions by computer calculations using the formula in Section 2 and write $\tilde{F}_{\gamma_{j}}(S \tau)$ as polynomial in the string functions. We check that

$$
\tilde{F}_{\gamma_{j}}(S \tau)=\frac{e(\operatorname{sign}(D) / 8)}{\sqrt{|D|}} \tau^{k} \sum_{l} c_{\gamma_{j}, \gamma_{l}} \tilde{F}_{\gamma_{l}}(\tau)
$$

To see this, it is helpful to replace the weight $\lambda$ in the string function $c_{\lambda}^{\Lambda}$ in the expression of $\tilde{F}_{\gamma_{j}}(S \tau)$ and of the $\tilde{F}_{\gamma_{l}}$ by the unique dominant weight in the $\hat{W}$-orbit of $\lambda$ in the set of weights of $L(\Lambda)$. This shows that $\tilde{F}_{\gamma_{j}}$ and the $\tilde{F}_{\gamma}$ have the desired transformation behaviour under $S$.

Since the theta function of a lattice transforms under the dual Weil representation of the corresponding lattice it is now obvious that the character

$$
\chi_{V}=\sum_{\gamma \in N^{\prime} / N} \tilde{F}_{\gamma} \vartheta_{\gamma}
$$

is invariant under $\mathrm{SL}_{2}(\mathbb{Z})$.

As in Section 3, let

$$
f(\tau)=\frac{1}{\eta(\tau)^{m} \eta(p \tau)^{m}}
$$

and define $T$-invariant functions $g_{j}$ by

$$
f\left(\frac{\tau}{p}\right)=g_{0}(\tau)+g_{1}(\tau)+\cdots+g_{p-1}(\tau)
$$

where $\left.g_{j}\right|_{T}(\tau)=e(j / p) g_{j}(\tau)$. Then

Theorem 5.7. The modular form $\tilde{F}$ is equal to the lift $F$ of the modular form $f$ to $N$.

Proof. Since the lattices $N$ and $\Lambda_{p} \oplus \sqrt{p} I_{1,1}$ have the same signature modulo 8 and isomorphic discriminant forms the components of $F=\sum F_{\gamma} e^{\gamma}$ 
are given by

$$
F_{\gamma}= \begin{cases}f+g_{0} & \text { if } \gamma=0 \\ g_{j} & \text { if } \gamma \neq 0 \text { and } \gamma^{2} / 2=-j / p \bmod 1\end{cases}
$$

We calculate the first nonvanishing coefficient of the string functions by computer using Freudenthal's formula and determine the singular coefficients of $\tilde{F}$. It turns out that they are equal to the singular coefficients of $F$. Hence the difference of $\tilde{F}$ and $F$ is a holomorphic modular form of negative weight which is finite at $\infty$ and therefore must be 0 .

If explicit formulas for the string functions are available then Theorem 5.7 can also be proved in the following way. The modular properties of the string functions imply that $\tilde{F}_{\gamma}$ in the formula of Proposition 5.4 is a modular form of weight $k$ and some level $N$ with poles at cusps. The same is true for $F_{\gamma}$. Hence we can deduce equality of these functions by comparing sufficiently many coefficients. For $\hat{A}_{1}$ and $\hat{A}_{2}$, there are explicit formulas for the string functions determined by Kac and Peterson [15]. We have used them to verify the statement of Theorem 5.7 in the cases $p=2$ and 3 .

Unfortunately, explicit formulas for the string functions are known only in a very few cases. The advantage of the proof of Theorem 5.7 given above is that it only needs the first nonvanishing coefficient of the string functions which is easy to determine using Freudenthal's formula. Therefore our method can also be applied to the other prospective vertex operator algebras in [21].

\section{Construction of some generalized Kac-Moody algebras as bosonic strings}

We show that the physical states of a chiral bosonic string with vertex algebra $V \otimes V_{I I_{1,1}}$ give a realization of the generalized Kac-Moody algebra corresponding to the elements of order $p$ in $M_{23}$.

We assume now that the prospective vertex operator algebra $V$ in [21] with spin-1 algebra $\hat{A}_{p-1, p}^{r}$ exists and has a real form.

We will work in this section over the real numbers.

Let $V_{I I_{1,1}}$ be the vertex algebra of the Lorentzian lattice $I_{1,1}$. Recall that the $b, c$-ghost system of the bosonic string is described by the vertex superalgebra of the lattice $\mathbb{Z}$. It carries a conformal structure of weight -26 . We call the corresponding vertex operator superalgebra $V_{b, c}$. There is 
an action of the BRST-operator $Q$ with $Q^{2}=0$ on the vertex superalgebra

$$
V \otimes V_{I I_{1,1}} \otimes V_{b, c}
$$

The cohomology group of ghost number 1 has a Lie bracket [16] and we denote this Lie algebra by $G$. The vertex algebra $V$ is graded by the rational lattice $N^{\prime}$, so that $G$ is graded by $N^{\prime} \oplus I I_{1,1}$. The no-ghost theorem (cf. [2, Theorem 5.1]) implies that the graded dimensions are given by $\operatorname{dim} G_{\alpha}=2 m+2$ if $\alpha=0$ and $\operatorname{dim} G_{\alpha}=\left[F_{\alpha}\right]\left(-\alpha^{2} / 2\right)$ if $\alpha \neq 0$. Here $F_{\alpha}$ is the component of $F$ corresponding to $\alpha \bmod I I_{1,1}$ (cf. Theorem 5.7). Furthermore, Theorem 2 in [4] shows that $G$ is a generalized Kac-Moody algebra.

In order to obtain an even grading lattice $L$ we rescale $N^{\prime} \oplus I I_{1,1}$ by $p$. It is easy to see that $L$ has genus $I I_{2 m+1,1}\left(p^{+m}\right)$. The lattice $\Lambda_{p} \oplus I I_{1,1}$ has the same genus. It follows from Eichler's theory of spinor genera that there is only one class in this genus so that $L$ is isomorphic to $\Lambda_{p} \oplus I I_{1,1}$. Thus we have

Proposition 6.1. The Lie algebra $G$ is a generalized Kac-Moody algebra graded by the Lorentzian lattice $L$. The Cartan subalgebra has dimension $2 m+2$ and

$$
\operatorname{dim} G_{\alpha}= \begin{cases}{[f]\left(-\alpha^{2} / 2\right)} & \text { if } \alpha \in L \backslash p L^{\prime} \\ {[f]\left(-\alpha^{2} / 2\right)+[f]\left(-\alpha^{2} / 2 p\right)} & \text { if } \alpha \in p L^{\prime}\end{cases}
$$

for nonzero $\alpha$, where

$$
f(\tau)=\frac{1}{\eta(\tau)^{m} \eta(p \tau)^{m}}=q^{-1}+m+\cdots .
$$

We recall that $[f](n)$ denotes the coefficient at $q^{n}$ in the Fourier expansion of the function $f$.

Let $\rho$ be a primitive norm 0 vector in $I I_{1,1}$. Then $\rho$ is a Weyl vector for the reflection group $W$ of $L=\Lambda_{p} \oplus I I_{1,1}$ (cf. [1]).

Theorem 6.2. The denominator identity of $G$ is

$$
\begin{aligned}
& e^{\rho} \prod_{\alpha \in L^{+}}\left(1-e^{\alpha}\right)^{[f]\left(-\alpha^{2} / 2\right)} \prod_{\alpha \in p L^{\prime^{+}}}\left(1-e^{\alpha}\right)^{[f]\left(-\alpha^{2} / 2 p\right)} \\
& \quad=\sum_{w \in W} \operatorname{det}(w) w\left(e^{\rho} \prod_{n=1}^{\infty}\left(1-e^{n \rho}\right)^{m}\left(1-e^{p n \rho}\right)^{m}\right) .
\end{aligned}
$$


The real simple roots of $G$ are the simple roots of $W$, i.e., the norm 2 vectors in $L$ with $(\rho, \alpha)=-1$ and the norm $2 p$ vectors in $p L^{\prime}$ with $(\rho, \alpha)=-p$. The imaginary simple roots of $G$ are the positive multiples $n \rho$ of the Weyl vector with multiplicity $m$ if $p \nmid n$ and multiplicity $2 m$ if $p \mid n$.

Proof. We only have to prove the second statement. Let $K$ be the generalized Kac-Moody algebra with root lattice $L$, Cartan subalgebra $L \otimes \mathbb{R}$ and simple roots as stated in the theorem. We lift $f$ to a vector valued modular form $F$ on $L \oplus \sqrt{p} I_{1,1}$. Note that $F$ admits the same description as the lift of $f$ on $\Lambda_{p} \oplus \sqrt{p} I_{1,1}$ in Section 3. Then we apply the singular theta correspondence to $F$ to obtain an automorphic form $\Psi$ of singular weight. The expansion of $\Psi$ at any cusp is given by

$$
\begin{aligned}
e^{\rho} & \prod_{\alpha \in L^{+}}\left(1-e^{\alpha}\right)^{[f]\left(-\alpha^{2} / 2\right)} \prod_{\alpha \in p L^{\prime+}}\left(1-e^{\alpha}\right)^{[f]\left(-\alpha^{2} / 2 p\right)} \\
& =\sum_{w \in W} \operatorname{det}(w) w\left(e^{\rho} \prod_{n=1}^{\infty}\left(1-e^{n \rho}\right)^{m}\left(1-e^{p n \rho}\right)^{m}\right) .
\end{aligned}
$$

This is the denominator identity of $K$. We see that $G$ and $K$ have the same root multiplicities. The product in the denominator identity determines the simple roots of $G$ because we have fixed a Cartan subalgebra and a fundamental Weyl chamber. It follows that $G$ and $K$ have the same simple roots and are isomorphic.

\section{Appendix}

Below we list the groups $G$ as linear codes in $\mathbb{F}_{p}^{r}$ and orbit representatives of $G \backslash M$ together with their multiplicities.

In the case $p=2$, the glue code $G$ is the binary Hamming code of length 16 and orbit representatives are

- $(2,0)^{16}$

- $8 \times(1,1)^{16}$

- The remaining orbit representatives can be described as follows. In the dual binary Hamming code of length 16, for every codeword of weight 8 , identify the 1 -components with the highest weight $(1,1)$ and for the 0-components allow all combinations of $(2,0)$ and $(0,2)$ such that both of these highest weights appear an odd number of times. 
In the case $p=3$, the glue code $G$ is the ternary zero sum code of length 6 and orbit representatives are

- $(3,0,0)^{6}$

- $(1,1,1)^{4}(3,0,0)^{2}$ and all permutations

- $(2,0,1)^{5}(0,1,2)$ and $(2,1,0)^{5}(0,2,1)$

- $6 \times(1,1,1)^{6}$.

In the case $p=5$, the glue code is $\mathbb{F}_{5}^{2}$ and orbit representatives are

- $(5,0,0,0,0)^{2}$

- $(2,0,1,0,2)^{2}$

- $(2,0,0,2,1)(3,0,1,1,0)$ and $(3,0,1,1,0)(2,0,0,2,1)$

- $(1,1,1,1,1)(1,0,0,1,3)$ and $(1,0,0,1,3)(1,1,1,1,1)$

- $4 \times(1,1,1,1,1)^{2}$.

In the case $p=7$, the glue code is $\mathbb{F}_{7}$ and orbit representatives are

- $(7,0,0,0,0,0,0)$

- $(2,0,0,1,3,0,1)$ and $(2,1,0,3,1,0,0)$

- $(2,0,0,2,0,3,0)$

- $(1,0,1,0,1,2,2)$

- $3 \times(1,1,1,1,1,1,1)$.

\section{Acknowledgment}

We thank J. Fuchs, G. Höhn and C. Schweigert for helpful discussions and comments. We also thank the referee for suggesting many improvements to the paper.

\section{References}

[1] R.E. Borcherds, Lattices like the Leech lattice, J. Algebra 130 (1990), 219-234.

[2] R.E. Borcherds, The monster Lie algebra, Adv. Math. 83 (1990), 30-47. 
[3] R.E. Borcherds, Monstrous moonshine and monstrous Lie superalgebras, Invent. Math. 109 (1992), 405-444.

[4] R.E. Borcherds, A characterization of generalized Kac-Moody algebras, J. Algebra 174 (1995), 1073-1079.

[5] R.E. Borcherds, Automorphic forms with singularities on Grassmannians, Invent. Math. 132 (1998), 491-562.

[6] J.H. Conway and N.J.A. Sloane, Sphere packings, lattices and groups, 3rd edn., Grundlehren der mathematischen Wissenschaften, 290, Springer, New York, 1998.

[7] I.B. Frenkel, Y.-Z. Huang and J. Lepowsky, On axiomatic approaches to vertex operator algebras and modules, Mem. Amer. Math. Soc. 104 (1993).

[8] J. Fuchs, Simple WZW currents, Comm. Math. Phys. 136 (1991), $345-356$.

[9] J. Fuchs, Affine Lie algebras and quantum groups, Cambridge Monographs on Mathematical Physics, Cambridge University Press, Cambridge, 1995.

[10] I.G. Frenkel and Y. Zhu, Vertex operator algebras associated to representations of affine and Virasoro algebras, Duke Math. J. 66 (1992), 123-168.

[11] G. Höhn, Genera of vertex operator algebras and 3-dimensional quantum field theories, Vertex Operator Algebras in Mathematics and Physics, Fields Institute Communications, 39, Amer. Math. Soc., Providence, RI, 2003, 89-107.

[12] Y.-Z. Huang, Vertex operator algebras and the Verlinde conjecture, Proc. Natl. Acad. Sci. USA 102 (2005), 5352-5356.

[13] G. Höhn and N.R. Scheithauer, A natural construction of Borcherds' Fake Baby Monster Lie Algebra, Amer. J. Math. 125 (2003), 655-667.

[14] V. Kac, Infinite dimensional Lie algebras, 3rd edn., Cambridge University Press, Cambridge, 1990.

[15] V. Kac and D. Peterson, Infinite-dimensional Lie algebras, theta functions and modular forms, Adv. Math. 53 (1984), 125-264.

[16] B.H. Lian and G.J. Zuckerman, New perspectives on the BRSTalgebraic structure of string theory, Comm. Math. Phys. 154 (1993), 613-646. 
[17] P. Niemann, Some generalized Kac-Moody algebras with known root multiplicities, Mem. Amer. Math. Soc. 157 (2002).

[18] N.R. Scheithauer, Generalized Kac-Moody algebras, automorphic forms and Conway's group I, Adv. Math. 183 (2004), 240-270.

[19] N.R. Scheithauer, Moonshine for Conway's group, Habilitation thesis, Heidelberg, 2004.

[20] N.R. Scheithauer, On the classification of automorphic products and generalized Kac-Moody algebras, Invent. Math. 164 (2006), 641-678.

[21] A.N. Schellekens, Meromorphic $c=24$ conformal field theories, Comm. Math. Phys. 153 (1993), 159-185.

[22] Y. Zhu, Modular invariance of characters of vertex operator algebras, J. Amer. Math. Soc. 9 (1996), 237-302.

DESY THEORY GROUP

NotKestrasse 85

BUILDING 2A

22603 HAMBURG

Germany

E-mail address: thomas.creutzig@desy.de

UNIVERSITY OF MANNHEIM

Department of MATHEMATiCS

68131 MANNHEIM

GERMANY

E-mail address: aklauer@rumms.uni-mannheim.de

UNIVERSITY OF EDINBURGH

MaXwell Institute for Mathematical Sciences

SCHOOL OF MATHEMATiCs

James Clerk MaXwell Building

MAYFIELD ROAD

EDINBURGH EH9 3JZ

UK

E-mail address: nils.scheithauer@ed.ac.uk

RECEIVED JANuARY 11, 2007 
